NISSUNA UMANA INVESTIGAZIONE SI PUO DIMANDARE VERA SCIENZIA S'ESSA NON PASSA PER LE MATEMATICHE DIMOSTRAZIONI LEONARDO DA VINCI

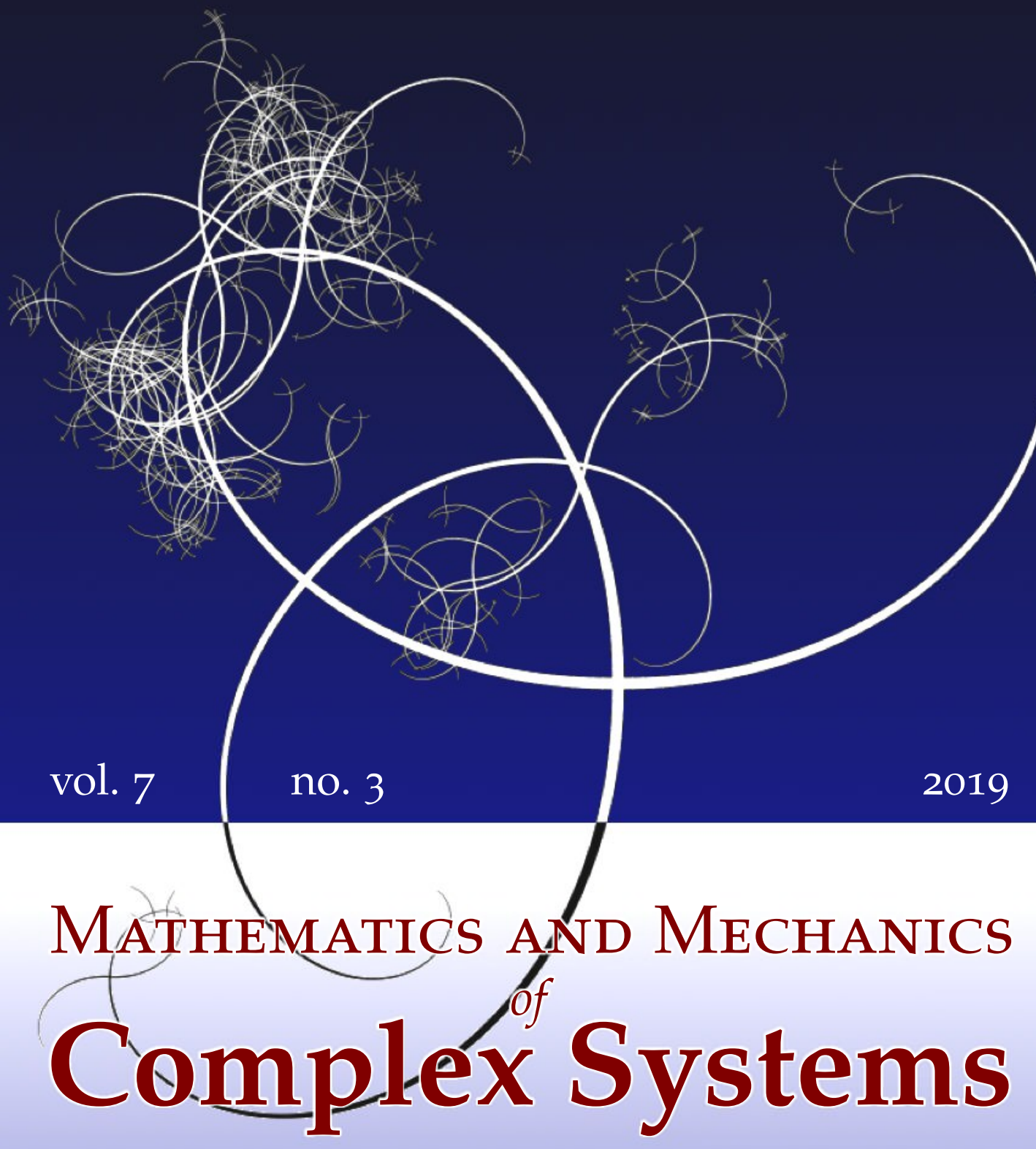

JUSTINE LOUIS

LOW-TEMPERATURE RATCHET CURRENT 


\title{
LOW-TEMPERATURE RATCHET CURRENT
}

\author{
JUSTINE LOUIS
}

We give an explicit expression for the low-temperature ratchet current in a multilevel system and find its numerical value as the number of states goes to infinity.

\section{Introduction}

In [Maes et al. 2014], the authors study stationary occupations in nonequilibrium multilevel systems at low temperatures. The system under consideration is an irreducible continuous time Markov jump process on a set of states $K$ with transition rates $\lambda(x, y)$ expressed in terms of the reactivities $a(x, y)$ and depending on the inverse temperature $\beta$. It is assumed that the system is in contact with an environment at uniform temperature and also subject to external forcing which breaks the Boltzmann occupation statistics. Being away from detailed balance implies that a current can flow in the system. To achieve nonequilibrium conditions, different physical models exist such as flashing ratchets. This corresponds to a random flipping between a flat potential and a nontrivial energy landscape. This is known as a continuous time Parrondo game [Parrondo 1998] and is studied in [Maes et al. 2014] as an example of their asymptotic formula for the stationary occupations. The authors model the multilevel system by a graph consisting of two rings of $N$ vertices which represent the states, and the edges stand for the preferred successors. This enables them to determine the direction of the ratchet current at low temperatures, which cannot be determined by entropic considerations only, and to evaluate it numerically while in the present paper we give an exact expression for it up to exponentially small corrections. This calculation confirms their result and in particular that the ratchet current is positive. The proof is based on an application of the Tutte matrix tree theorem. In the present section, we recall the definitions and results from [Maes et al. 2014, §3], and in the next section we give an explicit expression for the ratchet current and find its limit as the number of states goes to infinity.

\section{Communicated by Raffaele Esposito.}

The author acknowledges support from Swiss National Science Foundation grant 200021_132528/1. MSC2010: primary 05C30, 82C03; secondary 05C05, 05C20, 05C50.

PACS2010: 05.70.Ln.

Keywords: nonequilibrium statistical mechanics, direction current, Tutte matrix tree theorem. 
The zero-temperature logarithmic limit denoted by $\phi(x, y)$ is given by

$$
\phi(x, y):=\lim _{\beta \rightarrow \infty} \frac{1}{\beta} \log \lambda(x, y) .
$$

The zero-temperature logarithmic limit of the escape rates of state $x$ is denoted by $\Gamma(x)$ and given by

$$
\Gamma(x):=-\lim _{\beta \rightarrow \infty} \frac{1}{\beta} \log \left(\sum_{y} \lambda(x, y)\right)=-\max _{y} \phi(x, y) .
$$

The logarithmic-asymptotic transition probability is given by $e^{-\beta U(x, y)}$ where

$$
U(x, y):=-\Gamma(x)-\phi(x, y) .
$$

We have $U(x, y) \geqslant 0$ for all $x, y \in K$. The smaller $U(x, y)$ is, the larger is the probability of transition from state $x$ to state $y$. Hence, the set of preferred successors of $x$ is defined by

$$
\{y \in K \mid U(x, y)=0\} .
$$

When $U(x, y)=0$, the probability of transition from $x$ to $y$ is high. Thus, we consider the directed graph $K^{D}$ defined by the vertex set $K$ and edge set $\{(x, y) \mid$ $U(x, y)=0\}$ where $(x, y)$ indicates an oriented edge from $x$ to $y$. The transition rates are related to the reactivities by the relation

$$
\lambda(x, y)=a(x, y) e^{-\beta(\Gamma(x)+U(x, y))} .
$$

Using the Kirchhoff formula on $K^{D}$, the stationary occupation $\rho$ is given by

$$
\rho(x)=\frac{W(x)}{\mathscr{Z}}, \quad \text { where } W(x)=\sum_{\mathcal{T}_{x}} \prod_{(y, z) \in \mathscr{T}_{x}} \lambda(y, z) \text { and } \mathscr{E}=\sum_{x \in K} W(x)
$$

where the sum runs over all oriented edges $(y, z)$ in the in-tree $\mathscr{T}_{x}$. The lowtemperature asymptotic of the stationary occupation is given in:

Theorem [Maes et al. 2014, Theorem 2.1]. There is $\epsilon>0$ so that as $\beta \rightarrow \infty$,

with

$$
\rho(x)=\frac{1}{\mathscr{L}} A(x) e^{\beta(\Gamma(x)-\Theta(x))}\left(1+O\left(e^{-\beta \epsilon}\right)\right)
$$

$$
\begin{aligned}
& \Theta(x):=\min _{\mathcal{T}_{x}} U\left(\mathscr{T}_{x}\right) \quad \text { for } \quad U\left(\mathscr{T}_{x}\right):=\sum_{\left(y, y^{\prime}\right) \in \mathcal{T}_{x}} U\left(y, y^{\prime}\right), \\
& A(x):=\sum_{\mathcal{T}_{x} \in M(x)} \prod_{\left(y, y^{\prime}\right) \in \mathcal{T}_{x}} a\left(y, y^{\prime}\right)=e^{o(\beta)}
\end{aligned}
$$

where the last sum runs over all spanning trees minimizing $U\left(\mathscr{T}_{x}\right)$ (i.e., $\mathscr{T}_{x} \in M(x)$ if $\Theta(x)=U\left(\mathscr{T}_{x}\right)$ ). 


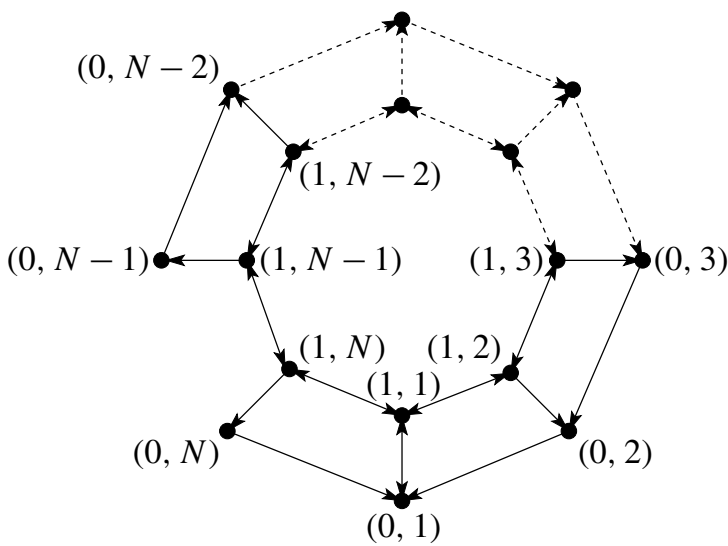

Figure 1. The directed graph $K^{D}$.

The case $a \gg 1$ satisfies detailed balance, and the model is then running on a single ring while in the case $a \ll 1$ the rings are uncoupled and detailed balance happens again for $a=0$. We thus consider the case $a=1$ corresponding to the nonequilibrium situation. The states, or energy levels, on the outer ring are denoted by $(0, i)$ and on the inner ring by $(1, i)$, where $i=1, \ldots, N$. The energies are denoted by $E_{i}, i=1, \ldots, N$, and are such that $E_{1}<\cdots<E_{N}$. The transition rates on the outer ring are thus given by

$$
\lambda((i, 0),(i+1,0))=e^{\beta\left(E_{i}-E_{i+1}\right) / 2}, \quad \lambda((i+1,0),(i, 0))=e^{\beta\left(E_{i+1}-E_{i}\right) / 2} .
$$

On the inner ring, the transition rates are constant and equal to one, that is,

$$
\lambda((i, 1),(i+1,1))=\lambda((i+1,1),(i, 1))=1 .
$$

The two rings are connected with transition rates constant and equal to one:

$$
\lambda((i, n),(i, 1-n))=1, \quad \text { where } n \in\{0,1\} .
$$

The digraph $K^{D}$ modeling the ratchet is represented in Figure 1. In the present case, for all $x \in K$, there exists an in-spanning tree $\mathscr{T}_{x}$ in $K^{D}$, so that $U\left(\mathscr{T}_{x}\right)=0$, and therefore, $\Theta(x)=0$. Let $\mathscr{D}$ be the set of states for which $\Gamma(x)=0$; it is given by $\mathscr{D}=\{(1,0),(i, 1), i=1, \ldots, N\}$. We denote $f \simeq g$ if $f=g+O\left(e^{-\beta \epsilon}\right)$ as $\beta \rightarrow \infty$. For $x \in \mathscr{D}$, we have $\rho(x) \simeq|M(x)| / \mathscr{L}$, where $|M(x)|$ is the number of in-spanning trees in $K^{D}$. For $x \notin \mathscr{D}$, the stationary distribution is exponentially small since from the theorem it is given by $\rho(x) \simeq|M(x)| e^{\beta \Gamma(x)} / \mathscr{W}$, with $\Gamma(x)<0$. The stationary ratchet current in the clockwise direction is given by

$$
J_{R}=j((i+1,0),(i, 0))+j((i+1,1),(i, 1)), \quad \text { for } i=1, \ldots, N,
$$

where $j(x, y)=\lambda(x, y) \rho(x)-\lambda(y, x) \rho(y)$. 
For $i=1$,

$$
J_{R}=j((2,0),(1,0))+j((2,1),(1,1))
$$

On the outer ring, $j((2,0),(1,0))=\lambda((2,0),(1,0)) \rho(2,0)-\lambda((1,0),(2,0)) \rho(1,0)$ with

$$
\begin{gathered}
\lambda((1,0),(2,0)) \simeq 0, \quad \lambda((2,0),(1,0))=e^{\left(E_{2}-E_{1}\right) \beta / 2}, \\
\rho(2,0) \simeq \frac{|M(2,0)|}{\mathscr{L}} e^{\beta \Gamma(2,0)}=\frac{|M(2,0)|}{\mathscr{L}} e^{-\left(E_{2}-E_{1}\right) \beta / 2},
\end{gathered}
$$

so that $j((2,0),(1,0)) \simeq|M(2,0)| / \mathscr{L}$.

On the inner ring, $j((2,1),(1,1))=\lambda((2,1),(1,1)) \rho(2,1)-\lambda((1,1),(2,1)) \rho(1,1)$ with

$$
\begin{gathered}
\lambda((2,1),(1,1))=\lambda((1,1),(2,1))=1, \\
\rho(2,1) \simeq \frac{|M(2,1)|}{\mathscr{Z}}, \quad \rho(1,1) \simeq \frac{|M(1,1)|}{\mathscr{Z}},
\end{gathered}
$$

so that $j((2,1),(1,1)) \simeq(|M(2,1)|-|M(1,1)|) / \mathscr{W}$. The ratchet current is thus given by

$$
J_{R} \simeq \frac{1}{\mathscr{E}}(|M(2,0)|+|M(2,1)|-|M(1,1)|) .
$$

Considering converging arborescences, the Laplacian matrix of a directed graph is defined by $L=D-A$ where $D$ is the diagonal out-degree matrix and $A=\left(A_{i j}\right)$ is the adjacency matrix such that $A_{i j}$ is the number of directed edges from $i$ to $j$. The rows and columns of $L$ are indexed by the vertices of the graph. Here, we index it first by the states on the outer ring and then the ones on the inner ring, that is $(1,0),(2,0), \ldots,(N, 0),(1,1),(2,1), \ldots,(N, 1)$. The Tutte matrix tree theorem [Aigner 2007] relates the number of spanning arborescences converging to $x$ in $K^{D}$ to the cofactors of the Laplacian $\operatorname{det} L_{x, y}$. Let $x \in K$. Then for all $y \in K$,

$$
|M(x)|=(-1)^{x+y} \operatorname{det} L_{x, y} .
$$

In particular, for $y=x$, we have $|M(x)|=\operatorname{det} L_{x}$. Therefore, we have

$$
J_{R} \simeq \frac{1}{\mathscr{L}}\left(\operatorname{det} L_{(2,1)}+\operatorname{det} L_{(2,0)}-\operatorname{det} L_{(1,1)}\right) .
$$

The Laplacian matrix is given by

$$
L=\left(\begin{array}{c|c}
F & G \\
\hline \mathrm{Id} & C
\end{array}\right)
$$


where $F$ is the $N \times N$ lower triangular matrix given by

$$
F=\left(\begin{array}{rrrrr}
1 & & & & \\
-1 & 1 & & & \\
& \ddots & \ddots & & \\
& & -1 & 1 & \\
-1 & & & 0 & 1
\end{array}\right),
$$

$G$ is the $N \times N$ matrix such that all coefficients are zero except $G_{(1,0),(1,1)}=-1$, the matrix Id is the $N \times N$ identity matrix, and $C$ is the circulant matrix

$$
C=\left(\begin{array}{rrrrr}
3 & -1 & & & -1 \\
-1 & 3 & \ddots & & \\
& \ddots & \ddots & \ddots & \\
& & \ddots & \ddots & -1 \\
-1 & & & -1 & 3
\end{array}\right) .
$$

\section{Calculation of the ratchet current}

From [Maes et al. 2014], the numerator of $J_{R}$ is given by

$$
\operatorname{det} L_{(2,1)}+\operatorname{det} L_{(2,0)}-\operatorname{det} L_{(1,1)}=\operatorname{det} B_{N-1}-2 \operatorname{det} B_{N-2}-2
$$

where $B_{N}$ is the $N \times N$ tridiagonal matrix with 3 on the diagonal and -1 on the two off-diagonals which satisfies the recurrence relation $\operatorname{det} B_{N}=3 \operatorname{det} B_{N-1}-$ $\operatorname{det} B_{N-2}$ with det $B_{1}=3$ and $\operatorname{det} B_{2}=8$. By solving the associated characteristic equation, we get

$$
\operatorname{det} B_{N}=\frac{5-3 \sqrt{5}}{10}\left(\frac{3-\sqrt{5}}{2}\right)^{N}+\frac{5+3 \sqrt{5}}{10}\left(\frac{3+\sqrt{5}}{2}\right)^{N} .
$$

The normalization factor is given by

$$
\mathscr{L}=\sum_{x \in K} \sum_{\mathscr{T}_{x}} \prod_{(y, z) \in \mathscr{T}_{x}} \lambda(y, z) \simeq \sum_{x \in \mathscr{D}}|M(x)|=\sum_{x \in \mathscr{D}} \operatorname{det} L_{x} .
$$

The sum is over the states in $\mathscr{D}$ since the contribution of the states which are not in $\mathscr{D}$ is exponentially damped. Therefore, we have

$$
\mathscr{Z} \simeq \operatorname{det} L_{(1,0)}+\sum_{i=1}^{N} \operatorname{det} L_{(i, 1)} .
$$

We have

$$
\operatorname{det} L_{(1,0)}=\operatorname{det} C \text {. }
$$


The circulant matrix $C$ has eigenvalues $\mu_{j}=3-2 \cos (2 \pi j / N), j=0,1, \ldots, N-1$ [Biggs 1993]. Hence,

$$
\operatorname{det} L_{(1,0)}=\prod_{j=0}^{N-1}(3-2 \cos (2 \pi j / N))=U_{N-1}^{2}(\sqrt{5} / 2)
$$

where $U_{N}$ is the $N$-th degree Chebyshev polynomial of the second kind. Thus,

$$
\operatorname{det} L_{(1,0)}=\left(\frac{3+\sqrt{5}}{2}\right)^{N}+\left(\frac{3-\sqrt{5}}{2}\right)^{N}-2 .
$$

From the Tutte matrix tree theorem, the cofactor $(-1)^{N+i} \operatorname{det} L_{(i, 1)}$ is equal to the number of converging arborescences to $(i, 1)$ and is equal to the cofactor of the Laplacian where row $(i, 1)$ and any column are removed. Since the only nonzero element of $G$ is in column indexed by $(1,1)$, we choose to remove that one, so that

$$
|M(i, 1)|=(-1)^{(N+i)+(N+1)} \operatorname{det} L_{(i, 1),(1,1)}=(-1)^{i+1} \operatorname{det} C_{(i, 1),(1,1)}
$$

since $F$ is lower triangular. On the other hand, by adding to the first column of $C$ all the other ones, we have

$$
\operatorname{det} C=\left|\begin{array}{rrrrr}
1 & -1 & & & -1 \\
1 & 3 & \ddots & & \\
& -1 & \ddots & \ddots & \\
& & \ddots & \ddots & -1 \\
1 & & & -1 & 3
\end{array}\right|=\sum_{i=1}^{N}(-1)^{i+1} \operatorname{det} C_{(i, 1),(1,1)}
$$

Putting (1), (2), (3), and (4) together, we have

$$
\mathscr{L} \simeq 2 \operatorname{det} C=2\left(\frac{3+\sqrt{5}}{2}\right)^{N}+2\left(\frac{3-\sqrt{5}}{2}\right)^{N}-4 .
$$

Up to exponentially small corrections $e^{-\beta \epsilon}$, the ratchet current is given for all $N$ by

$$
\begin{aligned}
J_{R} \simeq\left(\frac{5+3 \sqrt{5}}{10}\left(\frac{3+\sqrt{5}}{2}\right)^{N-1}+\frac{5-3 \sqrt{5}}{10}\left(\frac{3-\sqrt{5}}{2}\right)^{N-1}-\frac{5+3 \sqrt{5}}{5}\left(\frac{3+\sqrt{5}}{2}\right)^{N-2}\right. \\
\left.\quad-\frac{5-3 \sqrt{5}}{5}\left(\frac{3-\sqrt{5}}{2}\right)^{N-2}-2\right) /\left(2\left(\frac{3+\sqrt{5}}{2}\right)^{N}+2\left(\frac{3-\sqrt{5}}{2}\right)^{N}-4\right) .
\end{aligned}
$$

As a consequence, in the large system size limit the current saturates and has the limit

$$
\lim _{N \rightarrow \infty} J_{R} \simeq \frac{1}{2}-\frac{1}{\sqrt{5}}
$$




\section{Acknowledgements}

The author thanks Anders Karlsson for suggesting this problem to her. She also thanks the referee for useful comments.

\section{References}

[Aigner 2007] M. Aigner, A course in enumeration, Graduate Texts in Mathematics 238, Springer, 2007.

[Biggs 1993] N. Biggs, Algebraic graph theory, 2nd ed., Cambridge University, 1993.

[Maes et al. 2014] C. Maes, K. Netočný, and W. O. de Galway, "Low temperature behavior of nonequilibrium multilevel systems”, J. Phys. A Math. Theor. 47:3 (2014), 035002.

[Parrondo 1998] J. M. R. Parrondo, "Reversible ratchets as Brownian particles in an adiabatically changing periodic potential”, Phys. Rev. E 57:6 (1998), 7297-7300.

Received 8 Jul 2019. Revised 12 Aug 2019. Accepted 24 Sep 2019.

JUSTINE LOUIS: justine.louis@unige.ch

Section de Mathématiques, Université de Genève, Geneva, Switzerland

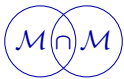


EDITORIAL BOARD

ANTONIO CARCATERRA

ERIC A. CARLEN

FRANCESCO DELL'ISOLA

RAFFAELE ESPOSITO

ALBERT FANNJIANG

Gilles A. FrancFort

Pierangelo Marcati

JEAN-JACQUES MARIGO

PETER A. MARKOWICH

MARTIN OSTOJA-STARZEWSKI

PIERRE SEPPECHER

DAVID J. STEIGMANN

PAUL STEINMANN

PierRe M. SuQueT

MANAGING EDITORS

MICOL AMAR

ANGELA MADEO

MARTIN OSTOJA-STARZEWSKI

\section{ADVISORY BOARD}

ADNAN AKAY

Holm AltenbaCH

MICOL AMAR

HARM ASKES

TEODOR ATANACKOVIĆ

VICTOR BERDICHEVSKY

GuY BouchitTÉ

ANDREA BRAIDES

ROBERTO CAMASSA

MAURO CARFORE

ERIC DARVE

FELIX DARVE

ANNA DE MASI

Gianpietro Del Piero

Emmanuele Di Benedetto

VICTOR A. EREMEYEV

BERNOLD FIEDLER

IRENE M. GAMBA

DAVID Y. GAO

SERGEy GaVRILYUK

Timothy J. HeAley

DOMINIQUE JEULIN

ROgER E. KHAYAT

CORRADO LATTANZIO

ROBERT P. LIPTON

ANGELO LUONGO

ANGEla MADEO

JUAN J. MANFREDI

CARlo MARCHIORO

ANIL MISRA

ROBERTO NATALINI

PATRIZIO NEFF

Thomas J. Pence

ANDREY PIATNITSKI

ERRICO PRESUTtI

MARIO PUlVIRENTI

Lucio Russo

Miguel A. F. SANJUAN

PATRICK SElvaduraI

MIROSLAV ŠILHAVÝ

GUIDO SWEERS

ANTOINETTE TORDESILLAS

LEV TRUSKINOVSKY

JUAN J. L. VELÁZQUEZ

VINCENZO VESPRI

ANGELO VULPIANI msp.org/memocs

Università di Roma "La Sapienza", Italia

Rutgers University, USA

(CO-CHAIR) Università di Roma "La Sapienza", Italia

(TREASURER) Università dell'Aquila, Italia

University of California at Davis, USA

(CO-CHAIR) Université Paris-Nord, France

Università dell' Aquila, Italy

École Polytechnique, France

DAMTP Cambridge, UK, and University of Vienna, Austria

(CHAIR MANAGING EDITOR) Univ. of Illinois at Urbana-Champaign, USA

Université du Sud Toulon-Var, France

University of California at Berkeley, USA

Universität Erlangen-Nürnberg, Germany

LMA CNRS Marseille, France

Università di Roma "La Sapienza", Italia

Université de Lyon-INSA (Institut National des Sciences Appliquées), France (CHAIR MANAGING EDITOR) Univ. of Illinois at Urbana-Champaign, USA

Carnegie Mellon University, USA, and Bilkent University, Turkey

Otto-von-Guericke-Universität Magdeburg, Germany

Università di Roma "La Sapienza”, Italia

University of Sheffield, UK

University of Novi Sad, Serbia

Wayne State University, USA

Université du Sud Toulon-Var, France

Università di Roma Tor Vergata, Italia

University of North Carolina at Chapel Hill, USA

Università di Pavia, Italia

Stanford University, USA

Institut Polytechnique de Grenoble, France

Università dell' Aquila, Italia

Università di Ferrara and International Research Center MEMOCS, Italia

Vanderbilt University, USA

Gdansk University of Technology, Poland

Freie Universität Berlin, Germany

University of Texas at Austin, USA

Federation University and Australian National University, Australia

Université Aix-Marseille, France

Cornell University, USA

École des Mines, France

University of Western Ontario, Canada

Università dell' Aquila, Italy

Louisiana State University, USA

Università dell'Aquila, Italia

Université de Lyon-INSA (Institut National des Sciences Appliquées), France University of Pittsburgh, USA

Università di Roma "La Sapienza", Italia

University of Kansas, USA

Istituto per le Applicazioni del Calcolo "M. Picone", Italy

Universität Duisburg-Essen, Germany

Michigan State University, USA

Narvik University College, Norway, Russia

Università di Roma Tor Vergata, Italy

Università di Roma “La Sapienza”, Italia

Università di Roma “Tor Vergata", Italia

Universidad Rey Juan Carlos, Madrid, Spain

McGill University, Canada

Academy of Sciences of the Czech Republic

Universität zu Köln, Germany

University of Melbourne, Australia

École Polytechnique, France

Bonn University, Germany

Università di Firenze, Italia

Università di Roma La Sapienza, Italia

MEMOCS (ISSN 2325-3444 electronic, 2326-7186 printed) is a journal of the International Research Center for the Mathematics and Mechanics of Complex Systems at the Università dell'Aquila, Italy.

Cover image: "Tangle” by $\odot$ John Horigan; produced using the Context Free program (contextfreeart.org).

PUBLISHED BY

7 mathematical sciences publishers

nonprofit scientific publishing

http://msp.org/

(C) 2019 Mathematical Sciences Publishers 
Mathematics and Mechanics of Complex Systems vol. 7 no. 3

Effective computation of $\mathrm{SO}(3)$ and $\mathrm{O}(3)$ linear representation symmetry classes

Marc Olive

Low-temperature ratchet current

Justine Louis

Eshelby's inclusion theory in light of Noether's theorem

Salvatore Federico, Mawafag F. Alhasadi and Alfio

Grillo

MEMOCS is a journal of the International Research Center for the Mathematics and Mechanics of Complex Systems at the Università dell' Aquila, Italy.

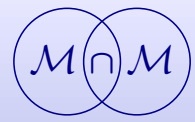

